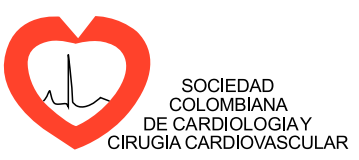

\title{
EDITORIAL
}

\section{Adultos con cardiopatías congénitas: una población creciente. Retos del presente y el futuro}

\author{
Néstor Sandoval
}

Cirugía cardiovascular, Instituto de cardiopatías congénitas, Fundación Cardioinfantil-Instituto de Cardiología, Bogotá, Colombia

Recibido el 3 de mayo de 2017; aceptado el 4 de mayo de 2017

Disponible en Internet el 30 de mayo de 2017

\section{Generalidades}

La población de adultos con cardiopatías congénitas es cada vez mayor y se estima que crece a un ritmo del $6 \%$ por año en países desarrollados. Sólo en Estados Unidos, se calcula que existen aproximadamente 1.4 millones de personas que padecen esta entidad, comparado con un millón de pacientes menores de 18 años con cardiopatía congénita.

Esto puede explicarse por la disponibilidad de métodos diagnósticos mejores y más oportunos, especialmente en el diagnóstico prenatal con eco fetal, resultados quirúrgicos e intervencionistas óptimos, cuidado intensivo cardiovascular especializado exitoso y la experiencia de centros de referencia de alto volumen, que brindan un manejo integral con menor mortalidad postoperatoria, lo cual conlleva mayor supervivencia a mediano y largo plazo. En general, con estas medidas se espera que el $85-90 \%$ de los pacientes con cardiopatías congénitas lleguen a la edad adulta ${ }^{1,2}$.

La expectativa de vida al nacer en el mundo ha mejorado, y en la actualidad en Colombia es de 73,95 años en la población general, 70,95 para los hombres y 77,10 para las mujeres, factor que favorece a los adultos con cardiopatías, pues se ven influidos por este cambio general ${ }^{3}$.

En un estudio llevado a cabo en Quebec se muestra como en dos periodos diferentes del 1987 a 1988 comparado con el periodo entre 2004 y 2005 , existe un gran cambio en la mortalidad de los niños con cardiopatías congénitas. En el primer año, la mortalidad fue del $25 \%$, comparado con la población general que era menor del 1\%. Posteriormente,

Correo electrónico: nsandoval@cardioinfantil.org en el segundo periodo estudiado del 2004 a 2005, esta mortalidad, especialmente en el primer año, disminuyó y llegó a ser muy similar a la de la población general, hecho que avala las hipótesis del porqué esta población llega en un porcentaje muy alto a la edad adulta ${ }^{4}$.

El estudio de Warnes et al., reportó cómo la posibilidad de sobrevida hasta la edad adulta luego de cirugía, ha cambiado desde los años 50 cuando esta era solo del $25 \%$, hasta el $90 \%$ para el $2010^{5}$ (fig. 1).

\section{Severidad de la enfermedad y causas de mortalidad}

Las cardiopatías congénitas tienen diferentes grados de severidad independientemente de la escala para determinar el riesgo quirúrgico (RACHS-1 o STAT) ${ }^{6,7}$. Algunos autores las clasifican en simples, moderadas y severas.

Las simples incluyen: comunicación interventricular simple, válvula aórtica bicúspide sin lesión, lesión mitral simple, ductus arterioso y estenosis pulmonar leve. Este grupo corresponde al $47 \%$ de las cardiopatías. El segundo grupo o moderado (que representa el 38\%), incluye cardiopatías como tetralogía de Fallot, defectos del septum interventricular, drenajes anómalos parciales, canal AV parcial, coartación de aorta, anomalía de Ebstein, estenosis pulmonar, entre otras. El tercer grupo es el de lesiones severas (15\%), que incluye algunas más graves como atresia mitral, transposición de grandes arterias, transposición corregida, síndromes de heterotaxia, doble salida ventricular, truncus arterioso, ventrículo único y lesiones cianóticas, además de síndrome de Eisenmenger ${ }^{8,9}$. 


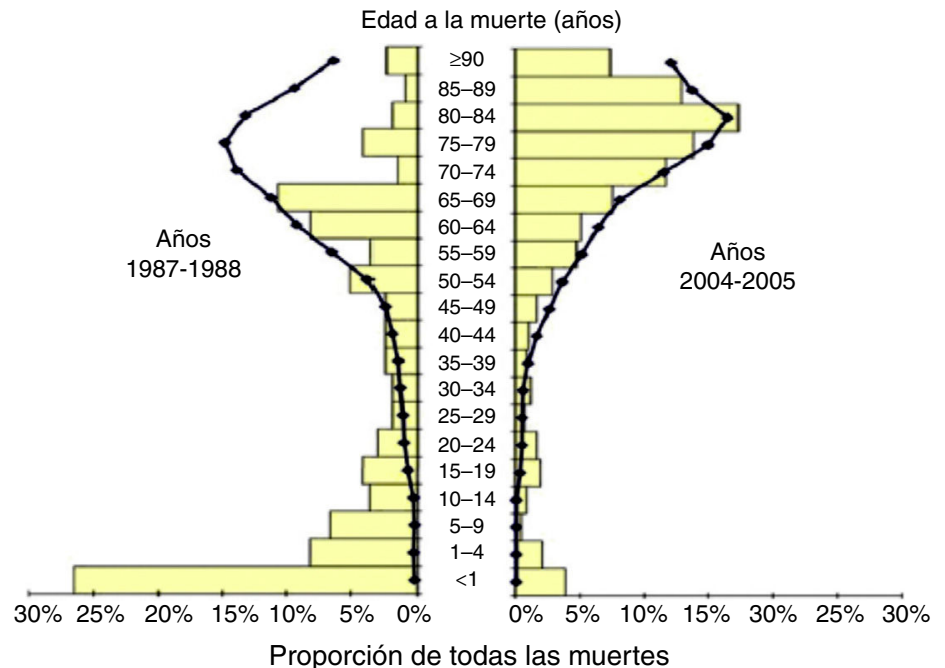

Figura 1 Distribución de pacientes con cardiopatía congénita por edad de fallecimiento en 1987-1988 y 2004-2005. Modificado de:Khairy et al. Changing mortality in congenital heart disease. JACC.2010;56:1149 -57.

En este estudio se muestran dos periodos diferentes del 1987 a 1988 comparado con el periodo entre 2004 y 2005 donde se aprecia el cambio en el riesgo de mortalidad, especialmente en el primer año de vida vs. la población general.

Lo más importante de esta clasificación es que incluye la dimensión de pronóstico. El $50 \%$ de los pacientes de los grupos moderado y severo requerirán de una reoperación y un gran número tendrá otras complicaciones como arritmias de difícil manejo, falla cardiaca, hipertensión pulmonar, etc.

La mortalidad se incrementa a una edad media de 48,8 años en cuyo caso esta mortalidad se debe en un $77 \%$ a enfermedad de origen cardiovascular y de estos el $45 \%$ a falla cardiaca $^{10}$. Igualmente, de acuerdo con la severidad de la enfermedad los más complejos morirán precozmente, entre estos pacientes con circulación de Fontan, cardiopatías complejas, Síndrome de Eisenmenger, corazón univentricular con ventrículo sistémico de característica derecha y anomalía de Ebstein?.

Otros estudios demuestran que las principales causas de morbimortalidad son la falla cardiaca en un $28 \%$ y causas no cardiovasculares en un $23 \%$ como malignidad, neumonías, falla hepática, endocarditis y falla renal. Un 19\% tienen muerte súbita, un $18 \%$, otras alteraciones cardiacas como enfermedad coronaria diabetes, hipertensión, obesidad y estrés, y un $14 \%$ lesiones vasculares, como aneurisma cerebral y aneurisma aórtico ${ }^{10}$.

La presencia de hipertension pulmonar en un $6 \%$ afecta la supervivencia ${ }^{11}$.

\section{Lesiones neurológicas y sicológicas}

Las lesiones neurológicas antiguas y los nuevos episodios asociados a alteraciones metabólicas afectan de manera considerable la independencia de estos pacientes. De igual forma, se predice que hasta un $30 \%$ de estos pacientes tienen desórdenes sicológicos y siquiátricos secundarios a trauma repetitivo, experiencias antiguas, al igual que desórdenes de estrés postraumático ${ }^{12}$.

\section{Contracepción y reproducción}

La contracepción y consejería son muy importantes pues la incidencia de hijos de estos pacientes con lesiones cardiovasculares es mayor que la de la población normal; sin embargo, gran número de estos pacientes tienen hijos y sus madres en especial durante el embarazo pueden sufrir mayor riesgo de complicaciones como arritmia, falla cardiaca y eventos cardiovasculares, específicamente aquellas con síndrome de Eisenmenger, cirugía de Fontan y enfermedades cianosantes ${ }^{13}$.

\section{Prevalencia de cardiopatías congénitas en Colombia}

La prevalencia de pacientes con cardiopatía congénita se ha estabilizado en el mundo, y en concreto, en Colombia es de $9 / 1.000$ nacidos vivos ${ }^{14,15}$. En el país, según cifras del DANE, nacen cada año 645.550 (año 2015) ${ }^{3}$ niños, se asume que al año nacen 5.805 nuevos niños con cardiopatías congénitas. De estos pacientes nuevos, el 70\% (4.063 pacientes) requieren tratamiento quirúrgico o intervencionista durante el primer año de vida pues la mortalidad sin tratamiento es alta, casi del $50 \%$. Infortunadamente solo se intervienen cerca de 2.349 con cirugía y unos 1.600 con procedimientos intervencionistas (total 3.949), de los cuales tan solo 1.500 de un total de 4.063 menores de un año, tienen oportunidad de tratamiento, con lo cual deja aproximadamente 2.500 nuevos niños sin tratamiento y riesgo alto de mortalidad en este primer $a n ̃ o^{16}$. 
Pacientes con cardiopatías congénitas que llegan a la edad adulta

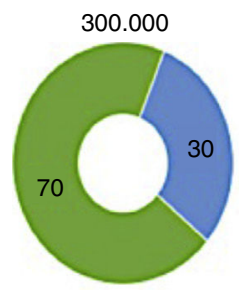

1965

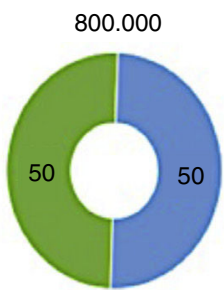

1985

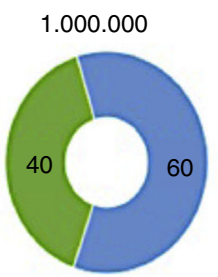

2005

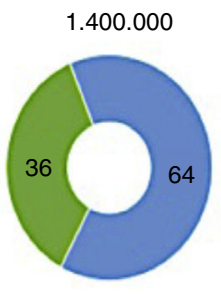

2015

Pediátricos

Adultos

Figura 2 Relación adultos y menores de 18 años con cardiopatías congénitas a través de los años desde los mediados de los años 60 cuando los adultos solo representaban el 30\% y el 2015 cuando se estima que sea del $64 \%$. Williams et al. J Am coll cardiol 2006 , Gilboa S circulation. 216; 134:101-109.

\section{Prevalencia de adultos con cardiopatía congénita}

Como se mencionó anteriormente, el porcentaje pacientes con cardiopatías congénitas que reciben tratamiento y sobreviven, ha mejorado de manera sobresaliente en el mundo y en Colombia, de modo que un mayor número de estos pacientes llegan a la edad adulta. Otro gran número de pacientes con enfermedades menos complejas sobrevivirá hasta la adultez y solo recibirán tratamiento luego de los 18 años, pero no se conocen datos.

Los estudios de prevalencia para adultos con cardiopatías congénitas se han hecho como revisión sistemática y con base en cálculos de evidencia donde se sugiere que la prevalencia de adultos con cardiopatías congénitas en regiones determinadas puede ser de 3.000 por millón de habitantes, con rangos entre 2.297 y $3.532^{12}$ o 4,09 adultos con cardiopatías congénitas por 1.000 adultos, según aproximaciones epidemiológicas fundamentadas en estudios de población de Quebec ${ }^{17,18}$. De acuerdo con estas aproximaciones, se estima que la relación adultos y menores de 18 años con cardiopatías congénitas, ha venido invirtiéndose desde mediados de los años 60, cuando los adultos solo representaban el 30\% y para el año 2015 sería mayor del 64\% ${ }^{2}$ (fig. 2).

\section{Adultos con cardiopatías congénitas en Colombia}

Según las aproximaciones mencionadas y si la cobertura y sobrevida de los recién nacidos fueran similares a las de países desarrollados, en Colombia se tendrían 141.000 adultos con cardiopatías congénitas. Sin embargo, debido al número de pacientes atendidos, a las condiciones propias del país, especialmente a la dificultad de la atención temprana del recién nacido, y a deficiencias del sistema se asume que se puede llegar a una falta de eficiencia en un $60 \%$, de ahí que tan solo el $40 \%$ llegará a la edad adulta, calculándose entonces un número aproximado de 56.400 adultos con cardiopatía congénita.

\section{Historia e impacto en el tratamiento}

La cirugía cardiaca inició a comienzos de los años cuarenta y cincuenta con la ligadura de ductus arterioso por Gross, en Boston, la fistula de Blalock-Taussig en el hospital John Hopkins en 1945, el reparo de coartación aórtica en 1944 en Suecia por Crafford y gran número de procedimientos cerrados. No obstante, con la aparición de la circulación extracorpórea introducida por Lillehey, Gibbon y Kirklin en los Estados Unidos a mediados de los años cincuenta se determinó un desarrollo espectacular en la cirugía intracardiaca, que luego de los años 80 se aplicó con éxito en el recién nacido y en los primeros meses de edad, cambiando el pronóstico y la sobrevida de estos pacientes.

Colombia, al igual que Latinoamérica, con el liderazgo de cirujanos y cardiólogos, no fue una excepción a este desarrollo y fue así como en 1948 se hizo el primer cierre de ductus, en 1952 Rueda realizó posiblemente el primer reparo de coartación aórtica y posteriormente un grupo de líderes nacionales en Bogotá y en Medellín iniciaron la cirugía intracardiaca con el empleo de la hipotermia moderada de superficie y el arresto cardiaco y más tarde con el uso de la circulación extracorpórea, siempre apoyados por pioneros internacionales como Walton Lillehey, Melrose y Humprey, quienes visitaron Colombia y ayudaron a desarrollar las diferentes estrategias del momento.

De igual forma, gracias a estos pioneros y a sus discípulos se crearon escuelas y centros especializados en cirugía cardiovascular, así como programas de entrenamiento de esta especialidad y por ello hoy los pacientes con cardiopatías congénitas en Colombia tienen mayor posibilidad de sobrevivir y llegar a la edad adulta ${ }^{19}$.

\section{Centros de cardiopatías congénitas para menores y adultos}

Los principales objetivos para el cuidado de estos pacientes se basan en los siguientes factores:

La importancia de mantener el cuidado a través de toda su vida. 


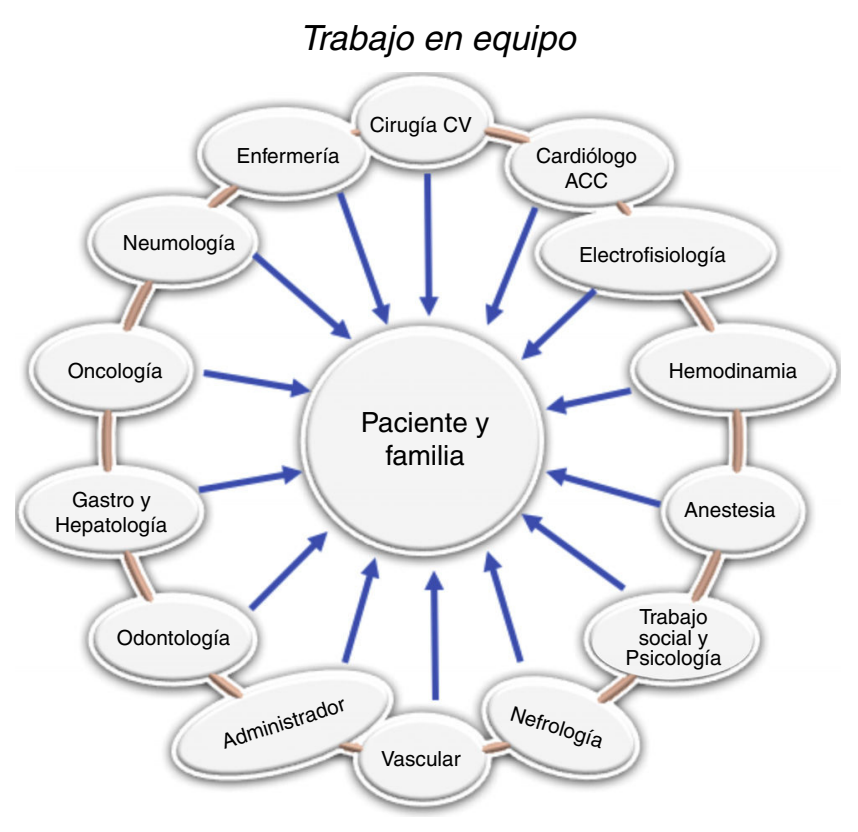

Figura 3 Centro de cardiopatías congénitas.

Grupo multidisciplinario que debe asumir el manejo integrado de un centro de adultos con cardiopatías congénitas.

La transferencia de los pacientes entre sus médicos pediatras a cardiólogos de adultos con la menor pérdida de su seguimiento.

La transición es un factor muy importante que incluye un proceso de maduración en el cual el paciente gana automanejo y logra crear algo de independencia del cuidado de su familia. Otros infortunadamente no lo logran por dificultades del neurodesarrollo o alteraciones sicosociales secundarias al proceso de tratamiento y sus posibles complicaciones y comorbilidades que desarrollan durante su vida.

Por todo lo descrito y el crecimiento constante de esta población se crearon los centros especializados en cardiopatías congénitas de los adultos. Estos deben incluir no solo un especialista en cardiopatías congénitas de los adultos, sino un grupo multidisciplinario para realizar el seguimiento y manejo (fig. 3). Los centros de referencia en general ven entre 2.500 y 3.000 pacientes al año y se sugiere la creación de un centro por cada 2.850 pacientes, hecho que con base en la proyección del número de adultos con cardiopatías congénitas en Colombia ameritaría tener al menos tres centros especializados en esta población.

La Fundación Cardioinfantil - Instituto de Cardiología es un centro de referencia en Colombia y Latinoamérica; desde el año 2005 al año 2016 se han intervenido quirúrgicamente un total de 444 adultos con cardiopatías congénitas de los cuales en su mayoría son defectos interauriculares e interventriculares, seguidos por reoperaciones en pacientes con reparo previo de tetralogía de Fallot y drenajes venosos parciales. La mortalidad de este grupo es baja de $1,6 \%$, pero la presencia de arritmias es común en el postoperatorio. El seguimiento muestra una supervivencia del $97 \%$ (IC $95 \%$ 84-97) a los 10 años $^{20}$.

\section{Conclusión}

La prevalencia de adultos con cardiopatías congénitas es creciente y esta población será uno de los grandes retos de la cardiología en el futuro.

Es crítico anticipar los cambios de estos pacientes con el tiempo y sus comorbilidades para minimizar las complicaciones a largo plazo.

La creación de centros de referencia será esencial para el manejo adecuado, la transición y transferencia que garanticen un proceso ordenado y ante todo no traumático para el paciente y su familia. En este sentido, es crucial el trabajo de un equipo multidisciplinario.

\section{Conflicto de intereses}

Los autores declaran no tener ningún conflicto de intereses.

\section{Bibliografía}

1. Moodle D, Adult congenital heart disease. Texas Heart Institute Journal. 2011;38:705-6.

2. Gilboa S, Devine OJ, Kucik JE, Oster ME, Riehle-Colarusso T, Nembhard WN, et al. Congenital heart defects in the United States. Estimating the magnitude of the affected population 2010. Circulation. 2016;134:101-9.

3. Dane, Colombia. Proyecciones anuales de población por sexo y edad 1985-2015. Disponible en: www dane.gov.co.

4. Khairy $\mathrm{P}$, Ionescu-Ittu R, Mackie AS, Abrahamowicz M, Pilote I, Marelli, AJ. Changing mortality in congenital heart disease. JACC. 2010;56:1149 -57.

5. Warnes CA, Liberthson R, Danielson GK, Dore A, Harris L, Hoffman IE, et al. Task Force 1: The Changing Profile of Congenital Heart Disease in Adult Life. JACC. 2001;37:1161-98.

6. Jenkins JJ, Gauvreau K, Newburger JW, Spray TL, Moller JH, lezzoni LI. Consensus-based method for risk adjustment for surgery for congenital heart disease. J Thorac Cardiovasc Surg. 2002;123:110-8.

7. O'Brien SM, Clarke DR, Jacobs JP, Jacobs ML, Lacour-Gayet FG, Pizarro C, et al. An empirically based tool for analyzing mortality associated with congenital heart surgery. J Thorac Cardiovasc Surg. 2009;138:1139-53.

8. Hoffman JIE, Kaplan S. The incidence of congenital heart disease. J Am Coll Cardiol. 2002;39:1890-900.

9. Diller GP, Kempny A, Alonso-Gonzalez R, Swan L, Uebing A, Li W, et al. Survival prospects and circumstances of death in contemporary adult congenital heart disease patients under follow-up at a large tertiary centre. Circulation. 2015;132: 2118-25.

10. Verheugt CL, Uiterwaal C, van der Velde TE, Meijboom FJ, Pieper PG, van Dijk APJ, et al. Mortaliy in adult congenital heart disease. Eur Heart J. 2010;31:1220-9.

11. Lowe BS, Therrien J, Ionescu-Ittu R, Pilote L, Martucci G, Marelli AJ. Diagnosis of pulmonary hypertension in the congenital heart disease adult population impact on outcomes. J Am Coll Cardiol. 2011;58:538-46.

12. Linda B, Pauliks. Depression in adults with congenital heart disease-public health challenge in a rapidly expanding new patient population. World J Cardiol. 2013;5:186-95.

13. Drenthen W, Pieper PG, Roos-Hesselink JW, van Lottum WA, Voors AA, Mulder JM, et al., On behalf of the ZAHARA Investigators. Outcome of Pregnancy in Women With Congenital Heart Disease. A literature review. J Am Coll Cardiol. 2007;49:2303-11. 
14. van der Linde D, Konings EEM, Slager MA, Witsenburg M, Helbing WA, Takkenberg J, et al. Birth prevalence of congenital heart disease worldwide. A Systematic review and meta-analysis. J Am Coll Cardiol. 2011;58:2241-7.

15. Zarante I, Franco L, López C, Fernández N. Frecuencia de malformaciones congénitas, evaluación y pronóstico de 52.744 nacimientos de tres ciudades colombianas. MEDICA. 2010;30:65-71.

16. Sandoval N, Kreutzer C, Jatene M, et al. Pediatric cardiovascular surgery in South America: current status and regional differences. World J Pediat Congenital Heart Surg. 2010;1:321.

17. van der Bom T, Bouma BJ, Meijboom FJ, Zwinderman AH, Mulder BJM. The prevalence of adult congenital heart disease, results from a systematic review and evidence based calculation. Am Heart J. 2012;164:568-75.

18. Marelli AJ, Therrien J, Mackie AS, Ionescu-Ittu R, Pilote L. Planning the specialized care of adult congenital heart disease patients: from numbers to guidelines; an epidemiologic approach. Am Heart J. 2009;157:1-8.

19. Laverde A. La cirugía cardiovasvular en Colombia. Rev Colomb Cardiol. 1985;1.

20. Sandoval N. Status and challenges of care for adults with congenital heart disease in Latin America. 5 th. Scientific meeting WSPCHS Adu Dhabi. UAE, Submitted WJPCHS. 2016 oct. 